\title{
Hybrid repair of a very late, post-aortic coarctation surgery thoracic aneurysm: a case report
}

\author{
Ioan Tilea ${ }^{1,2}$, Laszlo Hadadi ${ }^{3}$, Razvan C Serban ${ }^{3}$ and Brindusa Tilea ${ }^{2,4^{*}}$
}

\begin{abstract}
Introduction: Local aneurysms after surgical repair of coarctation of the aorta occur mainly in patients surgically treated by Dacron patch plasty during adulthood. The management of these patients is always problematic, with frequent complications and increased mortality rates. Percutaneous stent-graft implantation avoids the need for surgical reintervention.
\end{abstract}

Case presentation: We report a case involving the hybrid treatment by stent-graft implantation and transposition of the left subclavian artery to the left common carotid artery of an aneurysmal dilatation of the thoracic aorta that occurred in a 64-year-old Caucasian man, operated on almost 40 years earlier with a Dacron patch plasty for aortic coarctation. Our patient presented to our facility for evaluation with back pain and shortness of breath after minimal physical effort. A physical examination revealed stony dullness to percussion of the left posterior thorax, with no other abnormalities. The results of chest radiography, followed by contrast-enhanced computed tomography and aortography, led to a diagnosis of giant aortic thoracic aneurysm. Successful treatment of the aneurysm was achieved by percutaneous stent-graft implantation combined with transposition of the left subclavian artery to the left common carotid artery. His post-procedural recovery was uneventful. Three months after the procedure, computed tomography showed complete thrombosis of the excluded aneurysm, without any clinical signs of left lower limb ischemia or new onset neurological abnormalities.

Conclusions: Our patient's case illustrates the clinical outcomes of surgical interventions for aortic coarctation. However, the very late appearance of a local aneurysm is rather unusual. Management of such cases is always difficult. The decision-making should be multidisciplinary. A hybrid approach was considered the best solution for our patient.

Keywords: Aortic aneurysm, Aortic coarctation, Aortic stent-graft

\section{Introduction}

Coarctation of the aorta is a relatively frequent congenital cardiovascular disease, accounting for approximately $4 \%$ of all congenital cardiovascular abnormalities [1]. The usual approach is the surgical correction of the anomaly. Despite high initial success rates, more than 9\% of patients develop late complications, such as systemic hypertension, premature coronary artery disease, aortic

\footnotetext{
* Correspondence: brindusa_tilea@yahoo.com

${ }^{2}$ University of Medicine and Pharmacy of Targu Mures, 38 Gheorghe

Marinescu St, Targu Mures 540139, Romania

${ }^{4}$ Emergency Clinical County Hospital, Department of Infectious Diseases, 50

Gheorghe Marinescu St, 540136, Targu Mures, Romania

Full list of author information is available at the end of the article
}

valve abnormalities, aneurysm formation, or recoarctation $[2,3]$. Post-surgical aneurysms are described after subclavian flap angioplasty in $17 \%$ of cases, patch angioplasty in $14 \%$ of cases, interposition graft repair in $6 \%$ of cases and, occasionally, after end-to-end anastomosis in patients with persistent systemic hypertension $[4,5]$. Post-surgical aneurysms are particularly frequent after Dacron patch plasty performed in adulthood. Late occurrence of aortic aneurysms after surgical correction of aortic coarctation carries a significant risk of rupture and is associated with high mortality rates. Management of such patients is always challenging. Conservative treatment has unpredictable short-term results. A single-center study reported a $100 \%$ rate of rupture within 15 years [6]. Surgical 
reinterventions require a complex approach, including circulatory arrest, frequent use of blood products and a prolonged hospital stay. Moreover, surgical revision is associated with significant morbidity by paraplegia, injury of the central nervous system, or bleeding and 14\% inhospital mortality rates [7-9]. The use of balloonexpandable endovascular stents has been previously reported as successful in both primary treatment of coarctation and recoarctation of the aorta $[9,10]$. This technique also makes it possible to avoid a major surgical procedure and its inherent risks $[1,11,12]$.

Ince et al. reported no mortality and minimal morbidity in a series of six patients treated by percutaneous techniques and endovascular graft implantation for aneurysms occurring after previous repair of an aortic coarctation [1]. These results are significantly superior to those seen with open surgical treatment, which have $14 \%$ to $23.5 \%$ mortality rates $[6,13]$.

\section{Case presentation}

Almost two years ago, a 64-year-old Caucasian man was referred to our Cardiology Department for evaluation of back pain and shortness of breath on minimal physical activity. He had a long medical history, including surgical repair of aortic coarctation by Dacron patch plasty at the age of 26, with residual systemic hypertension. He also underwent bi-leaflet prosthetic valve (Sorin Biocarbon 27) implantation for severe degenerative stenosis of the bicuspid aortic valve at the age of 54. Twelve years previously he had been referred to hospital for ischemic stroke with left hemiparesis. On that occasion he was diagnosed as having a $5 \mathrm{~cm}$ diameter asymptomatic aneurysmal dilatation of the thoracic aorta at the site of the first surgical procedure. Given the lack of symptoms at that time, the decision was made to follow him with regular reassessments.

Twelve years later, given the onset of symptoms, he was hospitalized in our department. At admission, physical examination revealed stable vital signs, blood pressure at
$160 / 80 \mathrm{mmHg}$, normal heart rate at 64 beats per minute, stony dullness to percussion of the left posterior thorax, and no evidence of left ventricular dysfunction at rest. An electrocardiogram showed sinus rhythm, first-degree atrioventricular block with a PQ interval of 280 milliseconds, left bundle branch block and rare ventricular premature beats. Conduction abnormalities had been already documented during previous evaluations.

Initial evaluation consisted of a plain chest radiograph, which showed a well-contoured opacity with a diameter of approximately $10 \mathrm{~cm}$, next to the left border of the aorta (Figure 1A,B). The chest contrast-enhanced computed tomography (CT) scan confirmed the presence of a large dilatation of the thoracic aorta (Figure 2A,B). Transthoracic echocardiography found good left ventricular systolic function, normofunctional aortic prosthesis, no abnormalities of the native valves, absence of pulmonary hypertension, absence of pericardial effusion, and a dilated $(49 \mathrm{~mm})$ ascending aorta and aneurysmal descending thoracic aorta. Coronary angiogram and aortography performed in the same session showed no significant lesions of the epicardial vessels and location of the aortic aneurysm immediately below the left subclavian artery.

A multidisciplinary team, including a cardiologist, cardiovascular surgeon, angiographist and radiologist, decided on a hybrid resolution of the aneurysm.

One year after the last evaluation successful exclusion of the aneurysm was carried out by percutaneous stentgraft implantation (Proximal Free Flow Straight Valiant Thoracic $^{\circledR}$ TF3232C200X, Medtronic Inc., Minneapolis, MN, USA) using a right femoral approach (Figure 3A,B). The stent-graft partially covered the ostium of the left subclavian artery and a paraprosthetic leakage was observed (Figure 3C). The percutaneous intervention was continued with the surgical transposition of the left subclavian artery to the left common carotid artery within the same session. The post-procedural debranching had to be done because of the occlusion of the left subclavian artery during the procedure. Pre-procedural evaluation

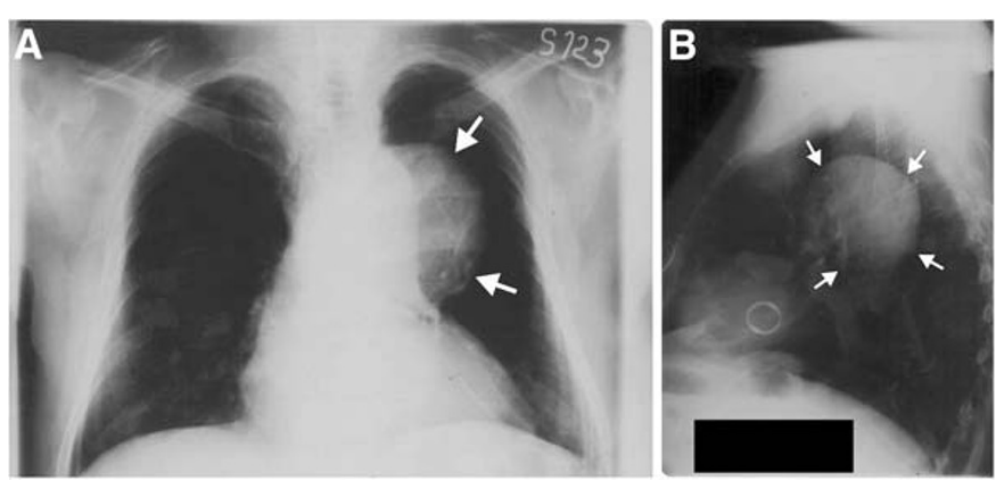

Figure 1 Chest radiography: antero-posterior (A) and left latero-lateral (B). 


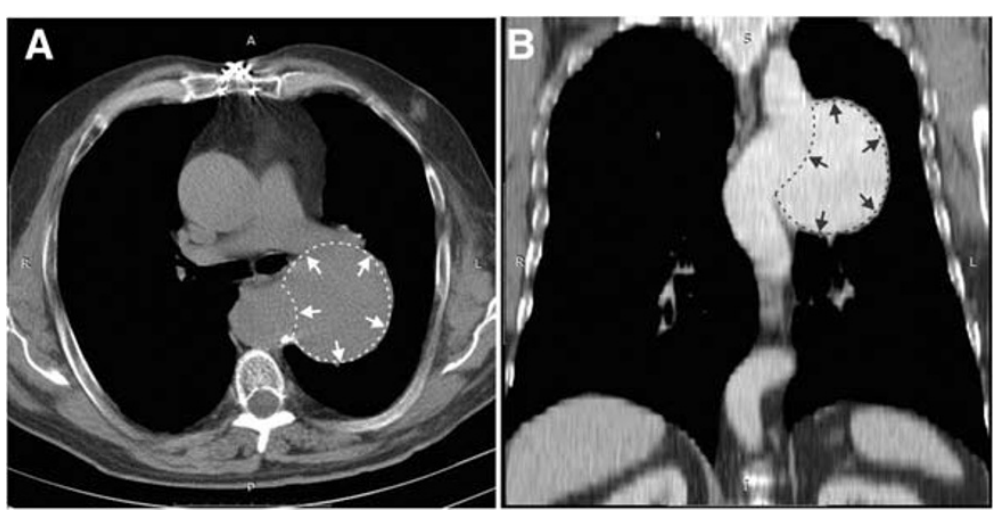

Figure 2 Contrast-enhanced computed tomography (CT) scan: transversal section (A) frontal plane bi-dimensional reconstruction (B). Image shows a $91 \times 80 \times 71 \mathrm{~mm}$ aneurysm of the descending aorta.

had not suggested this, but at the end of the interventional procedure the final angiogram revealed a partially covered ostium of the left subclavian artery by the stentgraft. The surgical possibilities for debranching in this situation were carotid-subclavian bypass or transposition of the left subclavian artery to the left common carotid artery. We chose the second procedure because of the presence of a paraprosthetic leakage originating from the left subclavian artery. The closure of the subclavian artery with sutures resolved both problems: the leakage and the left upper arm and vertebral artery vascularization. Both procedures were successful. Post-procedural evolution was uneventful, except for a fever of a few days duration of up to $38^{\circ} \mathrm{C}$, which was related to post-implantation syndrome, with no evidence of infection and mild residual dysphonia. Three months after the procedure, our patient was clinically well without any clinical signs of left lower limb ischemia or new onset neurological abnormalities. Contrast-enhanced CT revealed complete thrombosis of the excluded aneurysm (Figure 4A,B).

\section{Discussion}

We present a case that illustrates the clinical outcomes of surgical intervention for aortic coarctation: residual systemic hypertension, aortic valve disease requiring surgical intervention, and dilatation of the ascending aorta, as well as aneurysm formation at the site of the surgical anastomosis, all occurring after Dacron patch plasty. However, such a very late presentation and slow evolution of the local aneurysm are rather unusual. The management of such cases is always difficult, requiring a multidisciplinary approach. Such a large aneurysm is prone to rupture when conservatively treated, but a third surgical intervention would have been of particularly high risk. In this case, a multidisciplinary team decided on a hybrid approach, which confirmed once again the safety and feasibility of this procedure in high-risk patients. Thus, non-surgical reconstruction of late, post-aortic coarctation surgery thoracic aneurysms appears a safe and feasible technique. Interventional stent-graft placement allows avoiding repeated surgical interventions.

There are no clear guidelines, however, regarding the best treatment of aortic aneurysm occurring after surgical correction of aortic coarctation. Recent advances in surgical management offer better results from surgical repair in the hand of experts. However, such procedures cannot avoid relatively long circulatory arrest, frequent pulmonary complications, a need for blood products in

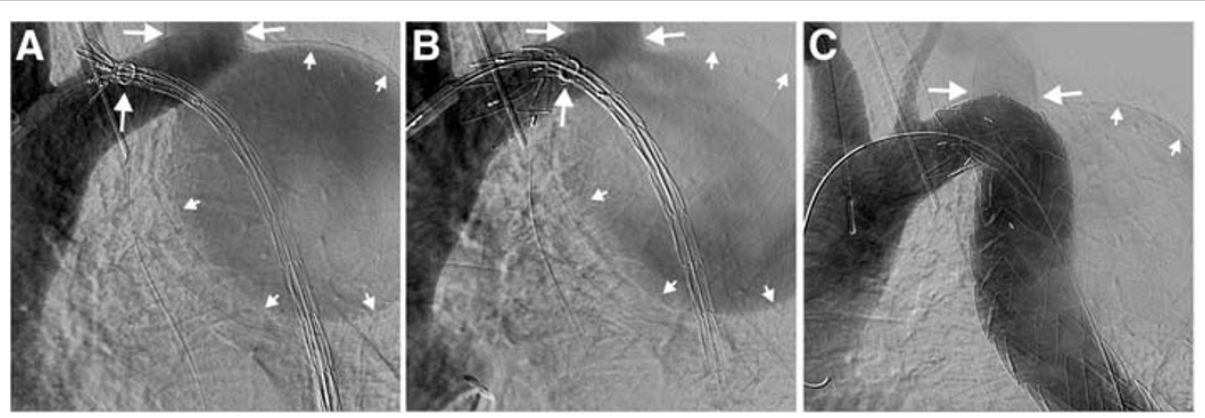

Figure 3 Angiography with stent-graft: positioning (A), and implantation (B). Immediate post-intervention angiography shows minimal leakage to the dilated left subclavian artery (C). 

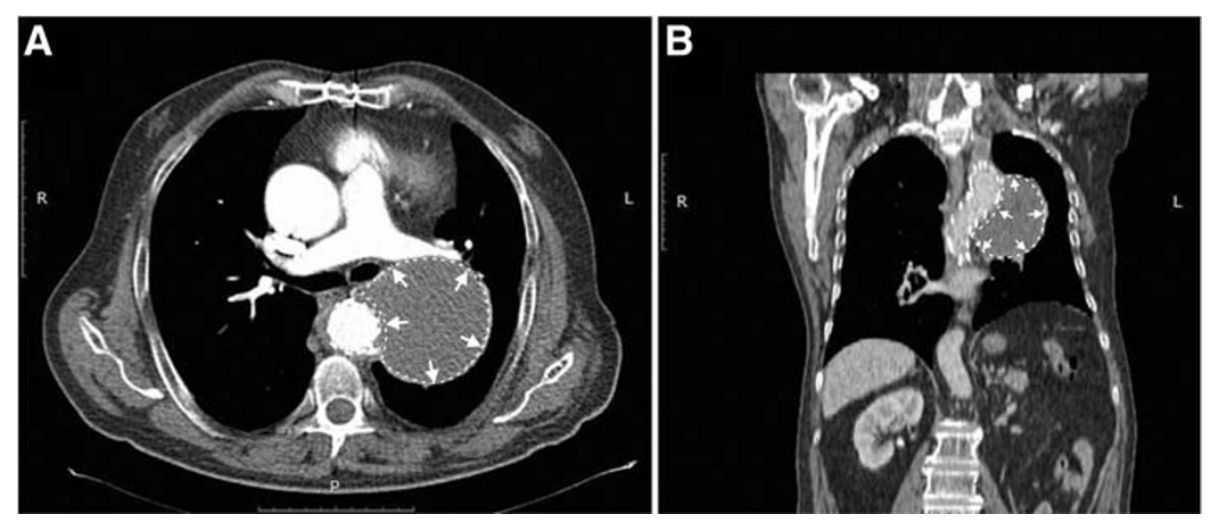

Figure 4 Three-month follow-up chest computed tomography (CT) scan: complete thrombosis of the aneurysm, without leakage from the stented segment. Transversal section (A), and frontal plane bi-dimensional reconstruction (B).

more than $50 \%$ of the cases and a prolonged hospital stay $[7,8]$. Conversely, percutaneous repair in such challenging cases requires less procedural time and no cardiac arrest. Thus, the interventional placement of stentgrafts offers the possibility of avoiding the risks associated with repeated surgery [1]. This case underscores the need for a multidisciplinary approach in the management of complex aortic diseases.

\section{Conclusions}

This case illustrates clinical outcomes of surgical interventions for aortic coarctation in a patient with the very late appearance of a local aneurysm. Clinical decisionmaking and treatment in this unusual situation should be multidisciplinary and a hybrid approach considered with percutaneous stent-graft implantation.

\section{Consent}

Written informed consent was obtained from the patient for publication of this case report and any accompanying images. A copy of the written consent is available for review by the Editor-in-Chief of this journal.

\section{Competing interests}

The authors declare that they have no competing interests.

\section{Authors' contributions}

IT performed the interventional procedure and drafted the manuscript. BT, RCS, and LH assured the follow-up of our patient, helped in drafting the manuscript and made critical revisions to the manuscript. All authors read and approved the final manuscript.

\section{Author details}

${ }^{1}$ Emergency Clinical County Hospital, Cardiac Catheterization Laboratory, 50 Gheorghe Marinescu St, Targu Mures 540136, Romania. ${ }^{2}$ University of Medicine and Pharmacy of Targu Mures, 38 Gheorghe Marinescu St, Targu Mures 540139, Romania. ${ }^{3}$ Emergency Clinical County Hospital, Cardiology Clinic I, 50 Gheorghe Marinescu St, Targu Mures 540136, Romania. ${ }^{4}$ Emergency Clinical County Hospital, Department of Infectious Diseases, 50 Gheorghe Marinescu St, 540136, Targu Mures, Romania.

Received: 23 November 2011 Accepted: 9 May 2012 Published: 30 August 2012

\section{References}

1. Ince H, Petzsch M, Rehders T, Kische S, Körber T, Weber F, Nienaber CA: Percutaneous endovascular repair of aneurysm after previous coarctation surgery. Circulation 2003, 108:2967-2970.

2. Toro-Salazar OH, Steinberger J, Thomas W, Rocchini AP, Carpenter B, Moller $\mathrm{J}$ : Long-term follow-up of patients after coarctation of the aorta repair. Am J Cardiol 2002, 89:541-547.

3. von Kodolitsch Y, Aydin MA, Koschyk DH, Loose R, Schalwat I, Karck M, Cremer J, Haverich A, Berger J, Meinertz T, Nienaber CA: Predictors of aneurysmal formation after surgical correction of aortic coarctation. J Am Coll Cardiol 2002, 39:617-624.

4. Parks WJ, Ngo TD, Plauth WH Jr, Bank ER, Sheppard SK, Pettigrew RI, Williams WH: Incidence of aneurysm formation after Dacron patch aortoplasty repair for coarctation of the aorta: long-term results and assessment utilizing magnetic resonance angiography with threedimensional surface rendering. J Am Coll Cardiol 1995, 26:266-271.

5. Aebert H, Laas J, Bednarski P, Koch U, Prokop M, Borst HG: High incidence of aneurysm formation following patch plasty repair of coarctation. Eur J Cardiothorac Surg 1993, 7:200-204.

6. Knyshov GV, Sitar LL, Glagola MD, Atamanyuk MY: Aortic aneurysms at the site of the repair of coarctation of the aorta: a review of 48 patients. Ann Thorac Surg 1996, 61:935-939.

7. Rokkas CK, Murphy SF, Kouchoukos NT: Aortic coarctation in the adult: management of complications and coexisting arterial abnormalities with hypothermic cardiopulmonary bypass and circulatory arrest. $J$ Thorac Cardiovasc Surg 2002, 124:155-161.

8. Gudbjartsson T, Mathur M, Mihaljevic T, Aklog L, Byrne JG, Cohn LH: Hypothermic circulatory arrest for the surgical treatment of complicated adult coarctation of the aorta. J Am Coll Cardiol 2003, 41:849-851.

9. Marshall AC, Perry SB, Keane JF, Lock JE: Early results and medium-term follow-up of stent implantation for mild residual or recurrent aortic coarctation. Am Heart J 2000, 139:1054-1060.

10. Ebheid MR, Prieto LR, Latson LA: Use of balloon-expandable stents for coarctation of aorta: initial results and intermediate-term follow-up. J Am Coll Cardiol 1997, 30:1847-1852.

11. Gawenda M, Aleksic M, Heckenkamp J, Krüger K, Brunkwall J: Endovascular repair of aneurysm after previous surgical coarctation repair. $J$ Thorac Cardiovasc Surg 2005, 130:1039-1043.

12. Kutty S, Greenberg RK, Fletcher S, Svensson LG, Latson LA: Endovascular stent grafts for large thoracic aneurysms after coarctation repair. Ann Thorac Surg 2008, 85:1332-1338.

13. Kieffer E, Bahnini A, Koskas F: Aberrant subclavian artery: surgical treatment in thirty-three adult patients. J Vasc Surg 1994, 19:100-109.

doi:10.1186/1752-1947-6-255

Cite this article as: Tilea et al:: Hybrid repair of a very late, post-aortic coarctation surgery thoracic aneurysm: a case report. Journal of Medical Case Reports 2012 6:255. 